\title{
Revealing potential long non-coding RNA biomarkers in lung adenocarcinoma using long non-coding RNA-mediated competitive endogenous RNA network
}

\author{
T-G. Zhu ${ }^{1}$, X. Xiao ${ }^{2}$, Q. Wei ${ }^{2}$, M. Yue ${ }^{3}$ and L-X. Zhang ${ }^{1}$ \\ ${ }^{1}$ Department of Pulmonary Disease, The Affiliated Hospital of Changchun University of Chinese Medicine, \\ Changchun, Jilin Province, China \\ ${ }^{2}$ Department of Heart Disease, The Affiliated Hospital of Changchun University of Chinese Medicine, Changchun, Jilin Province, China \\ ${ }^{3}$ Department of Internal Medicine, Lushuihe Forestry Bureau, Hospital of Jilin Province, Baishan, Jilin Province, China
}

\begin{abstract}
In our study, we aimed to reveal potential long non-coding RNAs (IncRNA) biomarkers in lung adenocarcinoma (LAD) using IncRNA-mediated competing endogenous RNAs (ceRNAs) network (LMCN). Competing IncRNA-mRNA interactions were identified using the hypergeometric test. Co-expression analysis for the competing IncRNA-mRNA interactions was implemented, and relying on the weight value $>0.8$, a highly competitive LMCN was further constructed. Degree distribution, betweenness and closeness for LMCN were carried out to analyze the network structure. Functional analyses of mRNAs in LMCN were carried out to further explore the biological functions of IncRNAs. Biclique algorithm was utilized to extract competing modules from the LMCN. Finally, we verified our findings in an independent sample set using qRT-PCR. Based on degrees $>60$, we identified 4 hubs, including DLEU2, SNHG12, HCP5, and LINC00472. Furthermore, 2 competing modules were identified, and LINC00472 in module 1 functioned as a hub in both LMCN and module. Functional implications of IncRNAs demonstrated that IncRNAs were related to histone modification, negative regulation of cell cycle, neuroactive ligand-receptor interaction, and regulation of actin cytoskeleton. qRT-PCR results demonstrated that IncRNAs LINC00472, and HCP5 were down-regulated in LAD tissues, while the expression level of SNHG12 was up-regulated in LAD tissues. Our study sheds novel light on the roles of IncRNA-related ceRNA network in LAD and facilitates the detection of potential IncRNA biomarkers for LAD diagnosis and treatment. Remarkably, in our study, LINC00472, HCP5, and SNHG12 might be potential biomarkers for LAD management.
\end{abstract}

Key words: Lung adenocarcinoma; Competing endogenous RNA; Long non-coding RNA; Hub; Diagnosis

\section{Introduction}

Lung adenocarcinoma (LAD) is the most common histological type of lung cancer, which is the leading cause of cancer-related deaths (1). An early and accurate diagnosis may warrant timely treatment to potentially decrease the mortality. However, a critical problem in the progression of LAD is the limited access to early detection and timely treatment. Therefore, understanding the mechanisms underlying LAD progression is urgent for improving the therapy and overall prognosis of this disease. However, traditional recognizable pathological symptoms have limited value in detecting early stage of LAD. Luckily, molecular biosignatures have been proven to be a promising tool for identifying patients in early-stage disease.

Long non-coding RNAs (IncRNAs), a major class of non-coding (ncRNAs), were determined as ncRNAs with more than 200 nucleotides in length (2). Growing evidence indicates that IncRNAs participate in a wide range of cellular processes, such as genomic imprinting, transcriptional and post-transcriptional regulation $(2,3)$. Significantly, IncRNAs have been implicated to participate in the development and progression of lung cancer. Known lung cancer-associated IncRNAs are few and include MALAT1 (LAD associated transcript) (4), and IncRNA GAS6-AS1 (5). Nevertheless, research about IncRNAs involved in $L A D$ is in its infancy. The identification of LAD-associated IncRNAs and the functions of IncRNAs require further investigation.

Typically, IncRNA functions are characterized using a 'guilt by association' strategy (6). It has been reported that IncRNAs harboring miRNA response elements (MREs) serve as competing endogenous RNAs (ceRNAs) to exchange with mRNAs via competing for common miRNAs (7). Experimental studies have demonstrated that aberrant expressions of important IncRNAs of ceRNA network

Correspondence: L. Zhang: <zhanglixiulx@163.com> 
have greater effects on the miRNA-regulated IncRNA/ mRNA ceRNA crosstalk interactions, thereby contributing to the occurrence and progression of cancers $(7,8)$. The IncRNA HULC plays an important regulatory role in lung cancer by acting as an endogenous ceRNA (9), revealing the functions of IncRNA-associated ceRNA crosstalk in lung cancer. Further, the dysregulated ceRNA network may provide new hope for exploring the pathogenesis of LAD and detecting new signatures with high accuracy in diagnosis.

In our study, with the goal of better understanding the molecular mechanisms underlying LAD, we aimed to reveal potential prognostic IncRNA biomarkers based on constructing a functional IncRNA-mediated ceRNA network (LMCN) involved in LAD.

\section{Material and Methods}

\section{Identifying miRNA-target interactions}

StarBase v2.0 (http://starbase.sysu.edu.cn/) was developed to systematically detect the RNA-RNA and proteinRNA interaction networks from 108 CLIP-Seq (PAR-CLIP, HITS-CLIP, iCLIP, CLASH) data sets, which provide highquality experimentally verified miRNA-target interactions manually curated from published studies. In our study, experimentally validated miRNA-mRNA interactions and IncRNA-miRNA interactions were downloaded from StarBase 2.0.

\section{Tissue samples and data collection}

The mRNA and IncRNA expression data of LAD were recruited from the research of $\mathrm{Xi}$ et al. (10) by repurposing the exon-array data on the Affymetrix Human 1.0 ST array from the ArrayExpress database (http://www.ebi.ac.uk/ arrayexpress/), which was accessible through E-GEOD12236. There were 40 samples in E-GEOD-12236, including 20 normal samples and 20 LAD samples. In detail, the probe sets were re-annotated to the human gene symbols, and 17,681 genes were identified. Then, the 17,681 genes were mapped to the miRNA-mRNA interactions and IncRNA-miRNA interactions. Ultimately, expression profiles of 10,485 mRNAs and 57 IncRNAs were identified. Afterwards, we, respectively, extracted the interactions containing any genes of 10,485 mRNAs and 57 IncRNAs from the miRNA-mRNA interactions and IncRNA-miRNA interactions. Totally, 334,014 miRNA-mRNA interactions and 695 IncRNA-miRNA interactions were identified.

\section{Detecting potential ceRNA interactions}

In an attempt to detect competing IncRNA-mRNA interactions, a hypergeometric test was employed in our study, which could evaluate the significance of the common miRNAs between each mRNA and IncRNA. The genome owned $K$ miRNAs, of which $M$ and $N$ were the counts of miRNAs related to the present mRNA and IncRNA, and $Y$ was shared miRNA number of IncRNA and
mRNA. With the goal of evaluating the enrichment significance of the shared miRNAs, the $P$ value was calculated using the following equation:

$$
P=1-\sum_{t=0}^{Y} \frac{\left(\begin{array}{c}
M \\
t
\end{array}\right)\left(\begin{array}{c}
K-M \\
N-t
\end{array}\right)}{\left(\begin{array}{l}
K \\
N
\end{array}\right)}
$$

Then, the original $P$ values were corrected using false discovery rate (FDR) according to Benjamini and Hochberg method (11). A FDR $<0.01$ was set as the cut-off criterion.

\section{Construction of a highly competitive LMCN}

$P C C$ is an index used to measure the co-expression probability of IncRNA-mRNA pairs. Specifically, PCC was counted according to the expression of the competing IncRNA-mRNAs pairs using the following formula:

$$
\mathrm{P}_{\mathrm{A}, \mathrm{B}}=\operatorname{cov}(\mathrm{A}, \mathrm{B}) / \sigma_{\mathrm{A}} \sigma_{\mathrm{B}}
$$

In this formula, $\operatorname{cov}(A, B)$ was the covariance of variable $\mathrm{A}$ and $\mathrm{B}$, while $\sigma_{\mathrm{A}}$ and $\sigma_{\mathrm{B}}$ respectively stood for the standard deviations (SD) for $A$ and $B$. The PCC absolute value of one interaction was defined as the weight value in our work, and only edges with correlations greater than the 0.8 were reserved to construct the LMCN. Cytoscape software (http://cytoscape.org/) was utilized to visualize the highly competitive LMCN.

\section{Centrality analysis for LMCN}

The centrality indexes are widely used for analyzing the properties of network, which contain degree, betweenness, closeness, and eigenvector centrality (12). Among these indexes, the degree is the simplest parameter. As reported, degree is defined as the quantity of links that a node connects with other nodes (13). Betweenness is an index of evaluating the influence of a node exerting over the spread of information through the network. A high betweenness denotes the significant roles of a node in information diffusion (14). Closeness is a measure of the mean length of the shortest paths to access all other proteins in the network (15). The network characteristics of LMCN, including degree centrality, betweenness centrality, and closeness centrality were analyzed using NetworkAnalyzer tool based on log-rank test. In our study, the nodes with degrees $>60$ were identified as hubs.

\section{Functional implication of IncRNAs}

Database for Annotation, Visualization and Integrated Discovery (DAVID) is a web tool providing a comprehensive set of functional annotation for researchers to understand the biological meaning behind a large number of genes (16). In our study, with the goal of investigating the biological functions of IncRNAs, we conducted gene oncology (GO) and pathway enrichment analysis for genes of LMCN using DAVID based on the 'guilt by 
association' strategy. In detail, we utilized Fisher's exact test to classify the functional terms. Then, $P$ values were adjusted relying on FDR according to Benjamini and Hochberg (11). GO terms with FDR $<0.01$ were considered statistically significant. Significant pathways were selected relying on FDR $<0.05$.

\section{Identifying synergistic, competing IncRNA modules}

Since the LMCN can offer a comprehensive view of all possible competing ceRNA interactions, which can be utilized to explore the regulatory characteristics of the IncRNAs, the sub-networks exhibited a more detailed picture of how the IncRNAs synergized with competing mRNAs. Biclique algorithm was recruited from the website of the Computational Biology Laboratory in the Department of Computer Science, lowa State University (http:// genome.cs.iastate.edu/supertree/download/biclique/). Previously, Biclique method was utilized in the study of ceRNA networks (17) to extract competing modules from the LMCN. This synergistic competing module is made up of a complete graph where an edge is realized from every vertex of an IncRNA set to every vertex of an mRNA set. In our study, the Biclique algorithm was employed to extract synergistic competing modules from LMCN.

\section{Total RNA extraction and $\mathrm{QRT}$-PCR verification}

We randomly selected 3 hub IncRNAs in the synergistic, competing IncRNA modules to verify the reliability of our results in LAD patients using qRT-pCR.

A total of 10 LAD patients were included in the current study, after informed consent forms were obtained. Tumor tissues were collected from these LAD patients as the experiment group, while the paired adjacent non-cancerous tissues were also obtained from the LAD patients as the control group. Samples were received with a quality assessment report confirming recruitment of tumor and adjacent non-tumor lung tissues.

Total RNA was isolated with RNA extraction kit (Invitrogen, USA) relying on the manufacturer's protocol. Reverse transcription reaction was implemented by means of $A 3500$ reverse transcription system kit (Promega, USA) according to the manufacturer's protocol. We used the qRT-PCR method to measure the expression levels of candidate IncRNAs according to the real-time PCR system (Applied Biosystems, USA). The qRT-PCR reaction was performed based on the following conditions: $95^{\circ} \mathrm{C}$ for $30 \mathrm{~s}, 40$ cycles of $95^{\circ} \mathrm{C}$ for $5 \mathrm{~s}$, and $60^{\circ} \mathrm{C}$ for $60 \mathrm{~s}$. Each sample was repeated in triplicate. All primers were purchased from Generay Biotech Co., Ltd. (China). The Ct-value for each sample was calculated with the $\Delta \Delta \mathrm{Ct}$ method (18), and fold change results were shown as $2^{-\Delta \Delta \mathrm{Ct}}$. The primer targeting beta-actin mRNA was used as internal control. All data were statistically compared using the paired $t$-test. The statistical analyses were conducted by means of the SPSS 21.0 (IBM, USA).

\section{Results}

\section{Identification of ceRNA interactions and construction} of a highly competitive LMCN

In order to extract potential IncRNA-mRNA competing pairs, a hypergeometric test was utilized to calculate the significance of the shared miRNAs between each IncRNAmRNA pair. As a result, a total of 57 IncRNAs, 10,133 mRNAs, and 48,939 ceRNA interactions were selected to establish an original network based on FDR 0.01, and their relationships were not displayed because the data in this original network could not be clearly visualized due to size. Then, PCC was used to measure the co-expression probability of the competing IncRNA-mRNAs pairs, and the absolute value of PCC difference of one interaction in two groups was defined as the weight value. The weight values of all the interactions in the original network are reported in Figure 1. Based on this figure, we found that the weight values ranged from 0.0004 to 1.29 . In our study, by applying a weight value $>0.8$, a high-competing LMCN was established. The LMCN contained 55 IncRNAs, 982 mRNAs, and 1104 ceRNA interactions, and is visualized in Figure 2A. From this figure, we found that the IncRNAs were in the central area of the network, but the mRNAs were typically in the outside layer. We discovered that a large proportion of mRNAs communicated with individual IncRNAs, and IncRNAs served as ceRNAs to connect with multiple mRNAs. These findings demonstrated that the aberrant expression of IncRNA ceRNA might lead to the extensive variation in gene expression through IncRNA-mRNA ceRNA crosstalk interactions, further implicating that ceRNA roles of IncRNAs is crucially important in LAD development and progression.

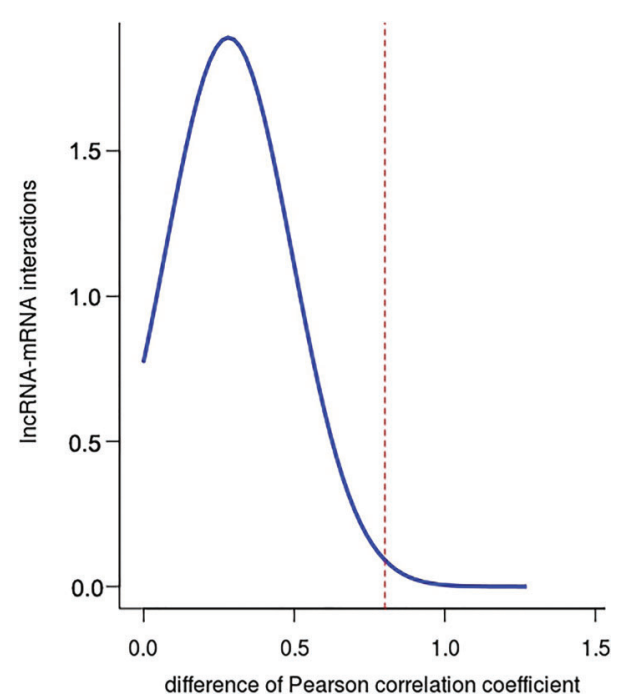

Figure 1. Weight distribution of 18,939 competitive endogenous RNAs (ceRNA) interactions. 

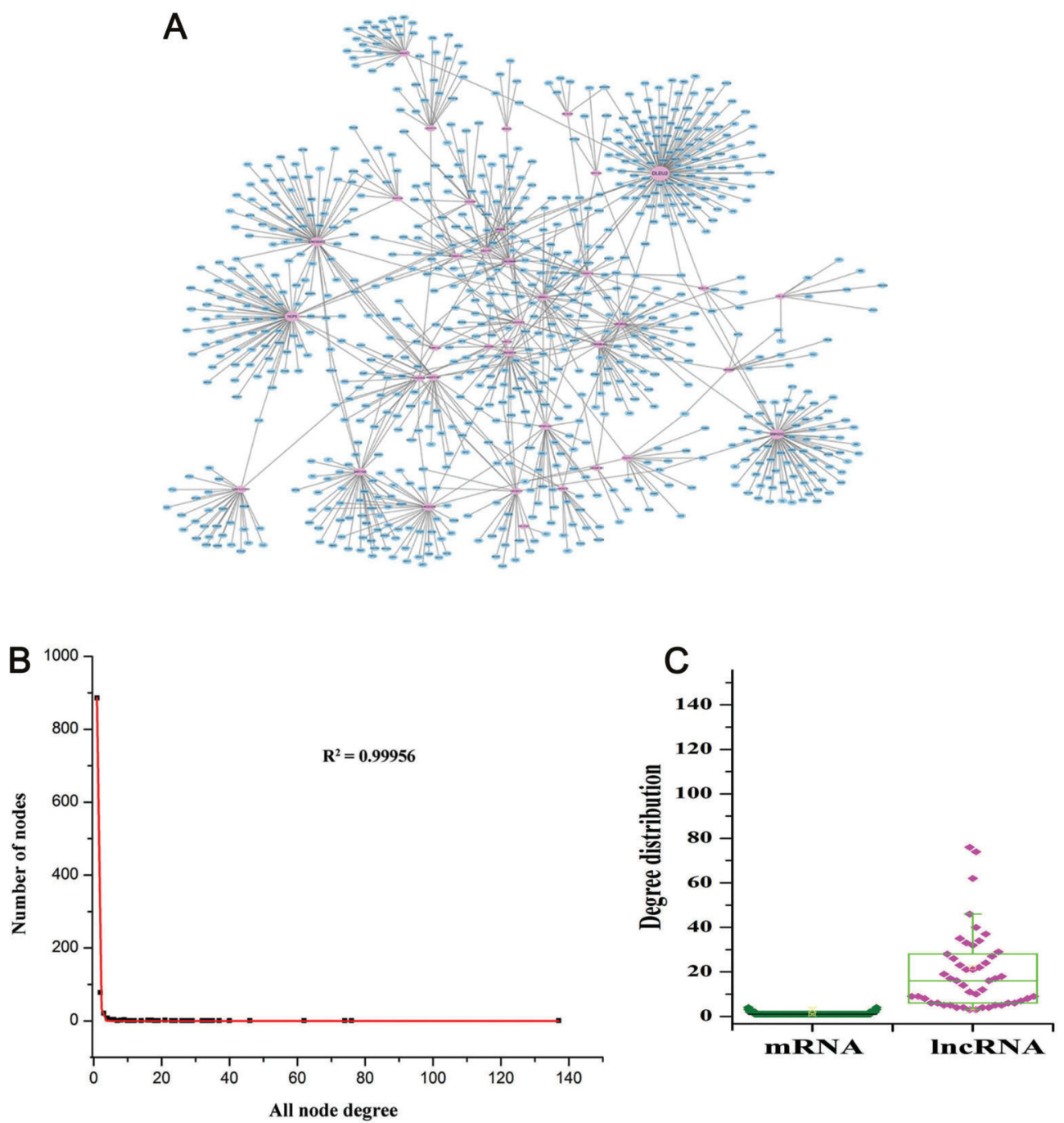

Figure 2. A, Highly competitive IncRNA-associated ceRNA network (LMCN). This network contained 55 IncRNAs, 982 mRNAs, and 1104 ceRNA interactions. Pink nodes denote IncRNAs, and blue nodes denote mRNAs. $B$, Degree analysis for the entire network reveals the specific properties of the LMCN. $C$, IncRNA are more critical components relative to mRNA ceRNAs in the LMCN. The IncRNAs had significantly higher degrees than mRNAs in the LMCN ( $t$-test, $P=2.49 E-23)$.

The original network (57 IncRNAs, 10,133 mRNAs, and 48,939 ceRNA interactions) is shown in Supplementary Table S1, and LMCN (55 IncRNAs, 982 mRNAs, and 1104 ceRNA interactions) is shown in Supplementary Table S2.

\section{Topological properties of the LMCN}

An investigation of the degree distribution of the entire network $\left(R^{2}=0.99956\right)$ suggested power law distributions (Figure 2B), which demonstrated that the LAD-associated LMCN was a scale-free network. These findings revealed that the LMCN was similar to other biological networks and was well organized by a core set of IncRNA-mRNA competing principles into structured rather than random networks. Generally, a higher degree indicated that the node was a hub, which involved more ceRNA interactions. These results uncovered that although the IncRNAs did not code for proteins, they exhibited more specific degree properties than mRNAs in the LMCN. To extract the hub nodes in the LMCN, all nodes in the LMCN were sorted in a descending order according to their degree distribution. Based on degrees $>60$, we identified 4 hub IncRNAs, including DLEU2 (degree=137), SNHG12 (degree=76), HCP5 (degree=74), and LINC00472 (degree=62).

Further, comparison analyses on the degree distribution, betweenness centrality, and closeness centrality between mRNAs and IncRNAs were carried out. IncRNAs had higher degree centrality $(P=2.49 E-23)$, betweenness 
centrality, and closeness centrality compared to mRNAs (Figures 2C, 3, and 4), implying that IncRNAs tended to be hub nodes.

\section{Functional implications of IncRNAs}

To further verify the potential functional implication of IncRNAs in LAD, we implemented functional enrichment analysis of mRNAs in the LMCN based on GO and KEGG pathways. Based on FDR $<0.01$, we found that the genes in the LMCN were significantly enriched in four GO terms, including histone modification ( $F D R=2.25 E-04)$, negative regulation of cell cycle $(\mathrm{FDR}=1.02 \mathrm{E}-03)$, stem cell population maintenance (FDR=6.09E-03), and maintenance of cell number (FDR=6.49E-03). Specific GO terms are shown in Figure 5. Moreover, we observed that the target genes of IncRNAs were remarkably involved in 14 pathways $(F D R<0.05)$, as listed in Table 1 . The top 3 pathways were respectively neuroactive ligand-receptor interaction, lysosome, and regulation of actin cytoskeleton. These results demonstrated that IncRNA-associated ceRNA regulation were involved in broad biological functions related to $L A D$.

\section{Identification of highly synergistic, competitive modules}

Subsequently, we further investigated the modularity feature of the LMCN. In order to extract synergistic, competing IncRNA modules, the Biclique algorithm was used in this study. In total, 2 synergistic, competitive modules comprising 32 genes were identified, as shown in Figure 6. These modules were numbered 1 and 2. We observed that these modules were the same in size with 16 genes. We also found that in module 1, a hub IncRNA LINC00472 functioned as a hub to compete with
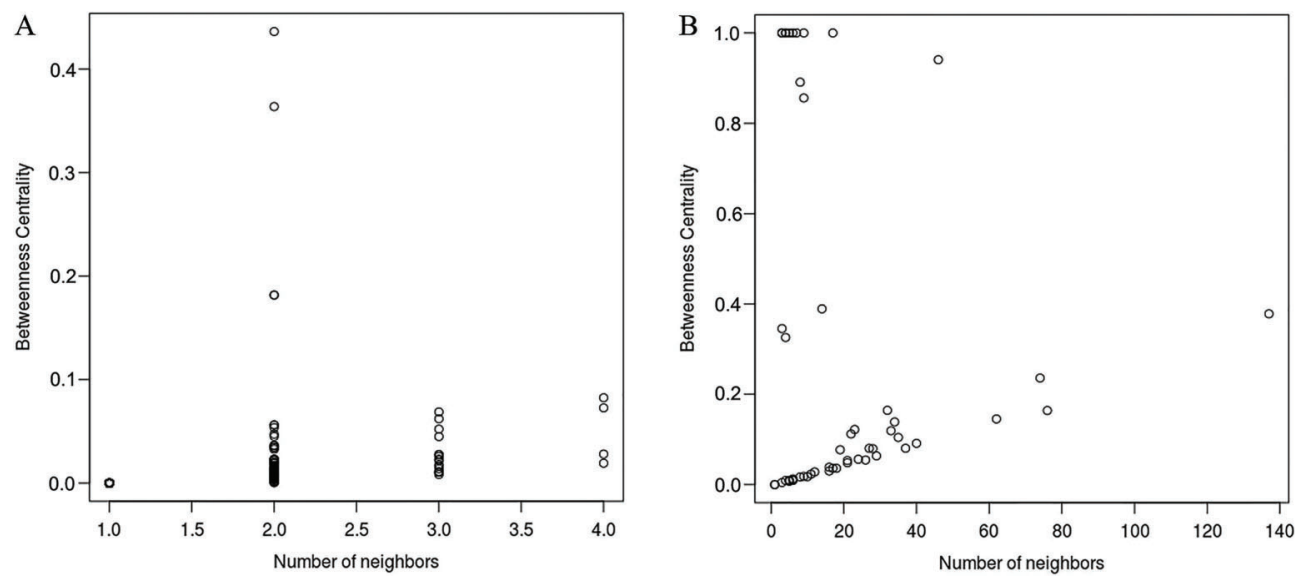

Figure 3. The IncRNA ceRNAs had a higher betweenness centrality than mRNA ceRNAs in the IncRNA-mediated ceRNAs network (LMCN). A, Betweenness distribution of mRNAs in the LMCN; $B$, Betweenness distribution of IncRNAs of the LMCN.
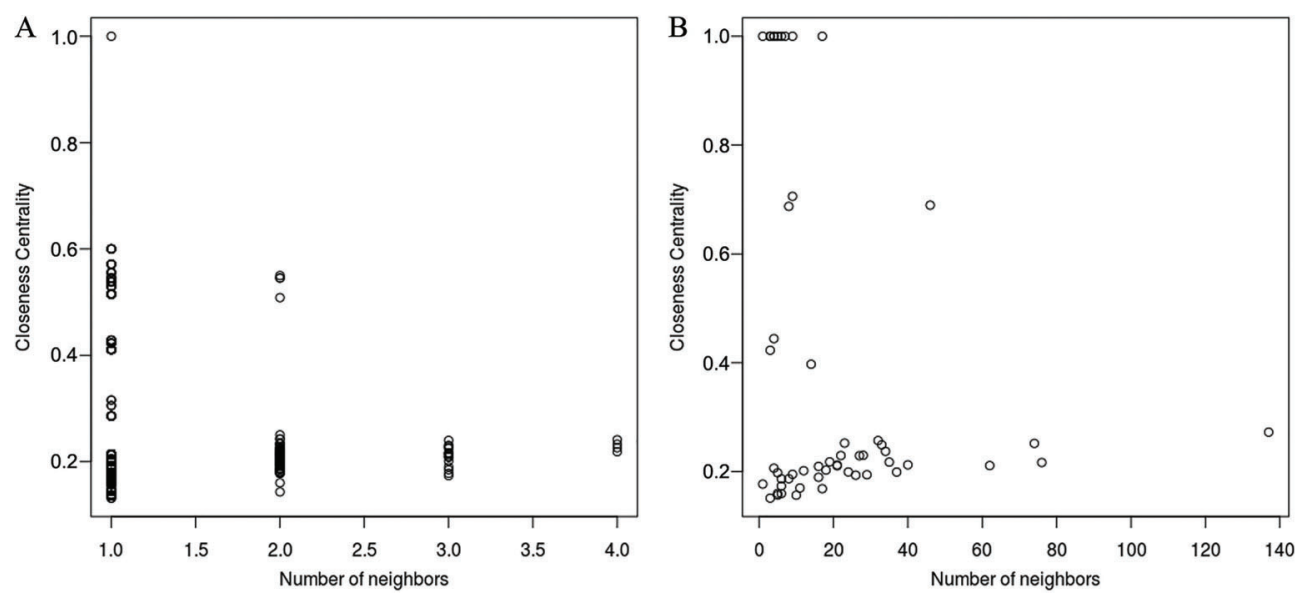

Figure 4. Comparison of closeness between IncRNAs and mRNAs in the IncRNA-mediated ceRNAs network (LMCN). A, Closeness distribution of mRNAs in the LMCN; $B$, Closeness distribution of IncRNAs of the LMCN. 


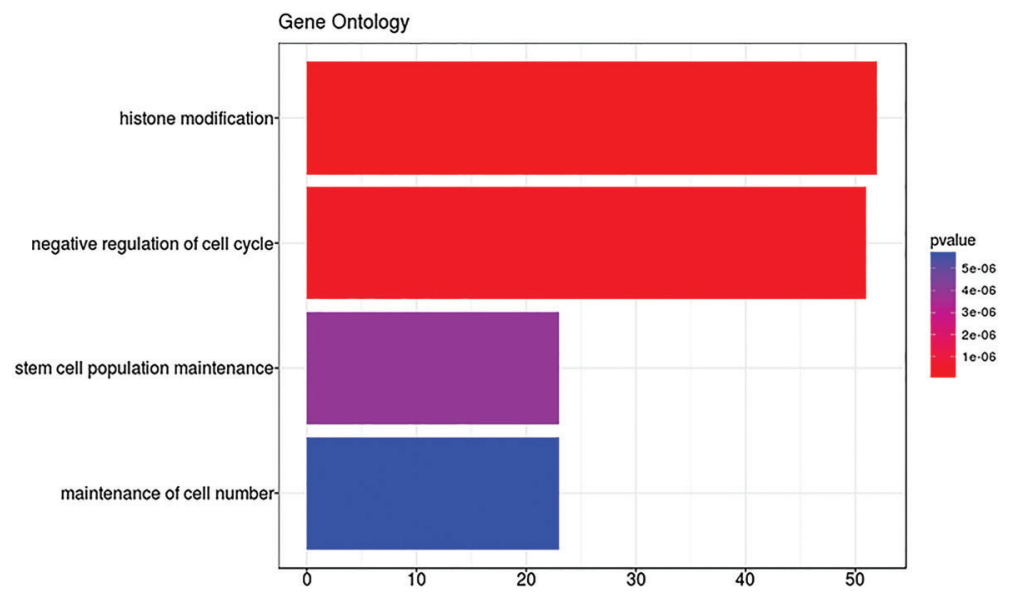

Figure 5. Significant gene oncology terms based on false discovery rate $<0.01$.

Table 1. Significant pathway terms based on false discovery rate $($ FDR $)<0.05$.

\begin{tabular}{ll}
\hline Pathway terms & FDR values \\
\hline Neuroactive ligand-receptor interaction & 0.01404349 \\
Lysosome & 0.01404349 \\
Regulation of actin cytoskeleton & 0.01404349 \\
p53 signaling pathway & 0.01404349 \\
Thyroid hormone signaling pathway & 0.01404349 \\
Prostate cancer & 0.01404349 \\
Pathways in cancer & 0.02321413 \\
Insulin signaling pathway & 0.03117087 \\
Acute myeloid leukemia & 0.03117087 \\
AMPK signaling pathway & 0.04013747 \\
Focal adhesion & 0.04013747 \\
B cell receptor signaling pathway & 0.04013747 \\
Colorectal cancer & 0.04013747 \\
Endometrial cancer & 0.04013747 \\
\hline
\end{tabular}

12 mRNAs (GATAD2B, MED13L, TSC22D1, SETD5, HIP1, ARNTL2, HMGN3, EPAS1, RELL1, ARHGEF10, ZNF532, and CTSS) and 3 IncRNAs (HCP5, HLA-F-AS1, and BAIAP2-AS1) in a 16-ceRNA module (Figure 6A), implying its important roles in LAD. These data also indicated that there were synergistic regulatory effects among these 4 IncRNAs. Within the other module (Figure 6B), LINC00649 competed with 7 mRNAs (KCNN4, STX1A, CCDC28A, SPTBN2, CDC5L. TMEM14A, and KATNA1) and 4 IncRNAs (LINC00094, MCM3AP-AS1, LINC00176, and SNHG12).

\section{qRT-PCR verification}

Eventually, we selected 3 hub IncRNAs (LINC00472, HCP5, and SNHG12) in the synergistic, competing IncRNA modules or LMCN to validate the reliability of the above analysis results. LINC00472 competed for many miRNAs, for example, hsa-miR-302e, hsa-miR-520e, hsa-miR-520a-3p,
hsa-miR-520b, hsa-miR-520c-3p, hsa-miR-520d-3p, hsamiR-372-3p, hsa-miR-373-3p, hsa-miR-302d-3p, hsa-miR302a-3p, hsa-miR-302c-3p, and hsa-miR-302b-3p. Another hub IncRNA HCP5 was found to compete for hundreds of miRNAs, such as hsa-miR-186-5p, hsa-miR-214-3p, hsa-miR-17-5p, hsa-miR-20a-5p, hsa-miR-519d-3p, hsamiR-653-5p, hsa-miR-93-5p, hsa-miR-106b-5p, hsa-miR20b-5p, and hsa-miR-106a-5p. Moreover, IncRNA SNHG12 competed for several miRNAs, including hsa-miR-181b$5 p$, hsa-miR-181a-5p, hsa-miR-16-5p, hsa-miR-15a-5p, hsa-miR-195-5p, hsa-miR-497-5p, hsa-miR-181c-5p, hsamiR-181d-5p, and hsa-miR-15b-5p. Specific information about miRNAs competed for by IncRNAs are shown in Supplementary Table S3.

The PCR results showed that LINC00472, and HCP5 were downregulated in LAD tissues when compared with adjacent non-tumor lung tissues, while SNHG12 was over-expressed in LAD tissues (Figure 7). The results from the $\mathrm{qRT}-\mathrm{PCR}$ validation further indicated that candidate IncRNAs including LINC00472, HCP5, and SNHG12 might play important roles in LAD progression, and further verified that our bioinformatics analysis was credible.

\section{Discussion}

Although some methods are available to support LAD diagnosis, for example, biopsy sampling and bronchoscopy, these methods are time-consuming, which may result in delays in early therapy. Thus, to ameliorate this condition, identifying cancer-related genes and the exact mechanisms of LAD occurrence and progression have received increasing attention. As documented, abnormal expression of IncRNAs has been broadly found in many diseases, and aberrant IncRNAs play important roles in biological functions of cancers. The functions of IncRNA are still not well known, and only very few have been well annotated. Hence, as a preliminary exploration for the underlying functional implications of the IncRNAs, 

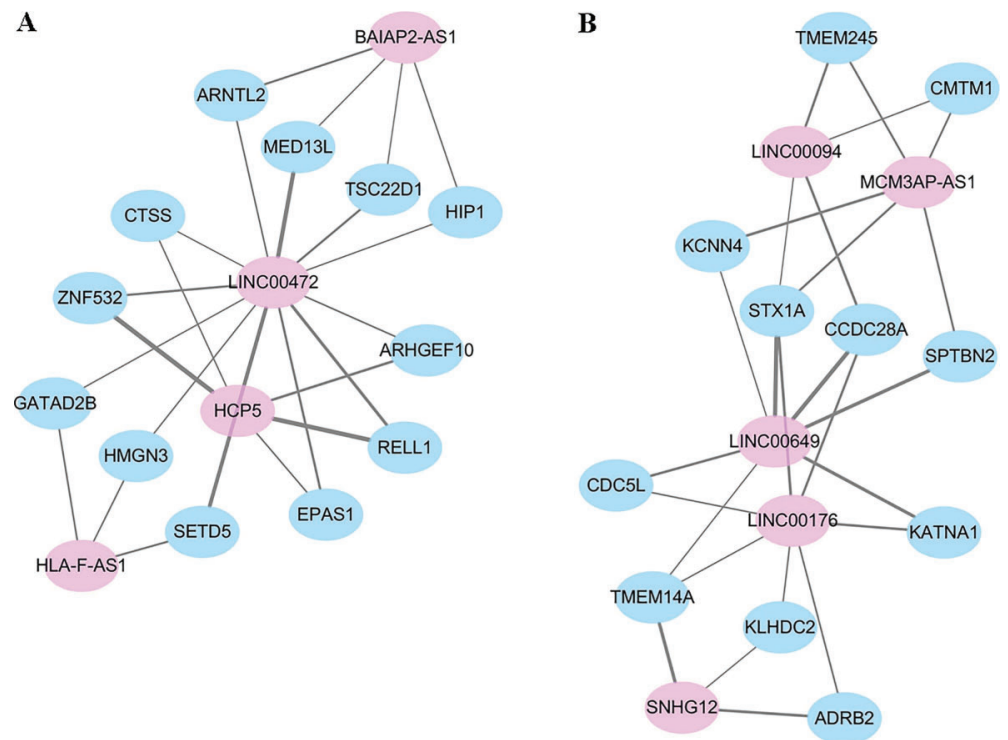

Figure 6. Extraction of synergistic, competing modules. A, Module 1. B, Module 2. Pink nodes denote IncRNAs, and blue nodes denote mRNAs.

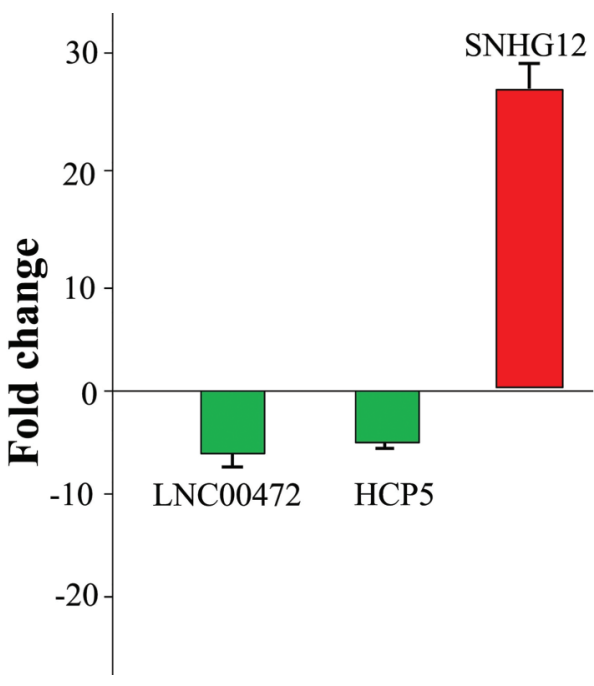

Figure 7. Fold change $\left(2-^{\Delta \Delta C t}\right)$ of 3 key IncRNAs by qRT-pCR. The primer targeting beta-actin mRNA was used as internal control. Green represents down-regulation, while red represents up-regulation. Data are reported as the mean fold-change $\pm S E$ of IncRNAs expression in tumor samples relative to normal samples.

'guilt by association' principle was used in our study to reveal biological processes mediated by the IncRNAs biomarkers based on functional enrichment analysis for mRNAs in the LMCN.

In the current study, we observed that mRNAs as ceRNA counterparts of IncRNA signatures were involved in four significant $G O$ terms. Histone modification was the most important $\mathrm{GO}$ term in our study. As we all know, histones constantly undergo post-translational modifications, for instance, methylation, acetylation, as well as phosphorylation. Of note, these modifications can modulate the nuclear chromatin setting and is a well-known epigenetic mechanism for regulating gene expression patterns (19). Histone modifications influence various cellular processes including chromatin modification, DNA replication and repair $(20,21)$. Importantly, the modification of histones has been demonstrated to be a predictor for colorectal cancer (22), and gastric carcinomas (23). Moreover, histone $\mathrm{H} 4$ modification has been indicated to play an important role in bronchial carcinogenesis and might be a candidate signature for therapeutic approaches in lung cancer (24). Accordingly, the GO term of histone modification might be closely associated with the LAD progression.

Negative regulation of cell cycle was another significant GO term. To our knowledge, the hallmark of cancer is uncontrolled cell proliferation caused by dysregulation of cell-cycle (25). Disturbed surveillance of cell-cycle progression, for example cellular evasion of cell-cycle checkpoints, which are driven by aberrant activation of cell-cycle regulators (cyclins and cyclin-dependent kinases), can cause the tumor development and progression (26). Activated cyclin-dependent kinase- 6 and cyclin-dependent kinase-2 during the G1-S cell-cycle transition are key regulators in the modifications of retinoblastoma pathway (27), disruption of which shows strong cell-proliferative activity and has been shown to play important roles in the pathogenesis of lung cancer (28). More importantly, Cai et al. (29) have reported that miR-186 exerts a tumorsuppressive role in the development of LAD, partially through regulation of cell-cycle progression. Hence, it is plausible that the IncRNA biomarkers act as ceRNAs involving the negative regulation of cell cycle, which might exert important functions in the occurrence and progression of LAD. 
Based on KEGG pathway analysis, neuroactive ligandreceptor interaction was the most significant pathway in the present study. The effect of a neuroactive steroid reveals a ligand-receptor interaction. Neuroactive steroids have been reported to influence the modulation of GABA receptor, and GABA receptors have been indicated to control cell proliferation (30). Cell proliferation is the hallmark of cancer. Further, growing body of evidence suggests that the actin-based processes, including cell polarity, filopodium formation, and cell migration, might be important in advanced cancers and may be associated with the formation of metastases (31). Thus, our results indicated that the key IncRNAs may exert important functions in initiation and progression of LAD through regulating significant pathways of neuroactive ligand-receptor interaction, and regulation of actin cytoskeleton.

According to the degree distribution, only 1 IncRNA (LINC00472) was a hub node in both LMCN and synergistic module in our study. LINC00472 has also been demonstrated to be abnormally expressed in epithelial ovarian cancer (32). In addition, a former study reported that LINC00472 over-expression significantly decreases the risk of relapse in patients with breast cancer, and LINC00472 also inhibits breast cancer cell proliferation and migration (33). In the current study, the expression level of LINC00472 was decreased in LAD compared to normal group. These results suggested that LINC00472 might play a crucial role in LAD as anti-onco-IncRNA.

HCP5 is named as HLA Class I Histocompatibility Antigen Protein P5, which is localized in the major histocompatibility complex (MHC) class I region. MHC regulates the immune responses relying on the presentation of tumor antigens (34). The regulation of tumor antigenspecific immune responses by an abnormal expression of MHC I molecules has been detected in a variety of cancers (35). Moreover, Liu et al. (36) have indicated that HCP5 is down-regulated in ovarian cancer. Consistent with this, HCP5 was down-regulated in LAD in our study.

\section{References}

1. Morgensztern D, Ng SH, Gao F, Govindan R. Trends in stage distribution for patients with non-small cell lung cancer: a National Cancer Database survey. J Thorac Oncol 2010; 5: 29-33, doi: 10.1097/JTO.0b013e3181c5920c.

2. Mercer TR, Dinger ME, Mattick JS. Long non-coding RNAs: insights into functions. Nat Rev Genet 2009; 10: 155-159, doi: $10.1038 / \mathrm{nrg} 2521$.

3. Fatica A, Bozzoni I. Long non-coding RNAs: new players in cell differentiation and development. Nat Rev Genet 2014; 15: 7-21, doi: $10.1038 / \mathrm{nrg} 3606$.

4. Ji P, Diederichs S, Wang W, Böing S, Metzger R, Schneider PM, et al. MALAT-1, a novel noncoding RNA, and thymosin $\beta 4$ predict metastasis and survival in early-stage non-small
Thus, we infer that IncRNA HCP5 might be involved in the pathogenesis of LAD.

SNHG12 is a member of small nucleolar RNA host genes (SNHGs), which have been indicated to contribute to cancer progression. Ruan et al. (37) have demonstrated that SNHG12 contributes to cell proliferation and migration. Of note, cell proliferation and migration are the main characteristics of cancer (25). Suppressing SNHG12 can cause the repression of cell proliferation, increased apoptosis, and G1 phase arrest in endometrial cancer (38). Remarkably, this IncRNA SNHG12 has been reported to be over-expressed in colorectal cancer (39) and breast cancer (40). In line with these results, our PCR results exhibited that SNHG12 was up-regulated in LAD, which confirmed that SNHG12 served as an onco-IncRNA in LAD. To the best of our knowledge, we provided the first evidence of SNHG12 dysregulation in LAD. In summary, we believe that over-expression of SNHG12 might promote the progression of LAD.

In conclusion, we constructed a LAD-specific IncRNAmediated ceRNA network, which enabled an overall view and analysis of IncRNA-associated ceRNA-regulated gene regulation in the progression of $L A D$ on a system-wide level. Our study will help to improve the understanding of IncRNAmediated ceRNA regulatory mechanisms in LAD progression and provide novel IncRNAs LINC00472, HCP5, and SNHG12 as candidate signatures for diagnosis and therapeutic targets. However, the biological roles of LINC00472, HCP5, and SNHG12 have not yet been determined by animal or patient experiments. Moreover, IncRNAs, miRNAs and mRNAs with differential expressions (between disease and control) might give much more information about potential biomarkers in LAD. In future work, we will construct a dysregulated $\mathrm{LMCN}$ to further detect the candidate IncRNA signatures involved in the pathogenesis of LAD.

\section{Supplementary material}

Click here to view [Excel] [pdf]. cell lung cancer. Oncogene 2003; 22: 8031-8041, doi: 10.1038/sj.onc. 1206928.

5. Han L, Kong R, Yin D-D, Zhang E-B, Xu T-P, De W, et al. Low expression of long noncoding RNA GAS6-AS1 predicts a poor prognosis in patients with NSCLC. Med Oncol 2013; 30: 1-7, doi: 10.1007/s12032-013-0694-5.

6. Wang P, Zhi H, Zhang Y, Liu Y, Zhang J, Gao Y, et al. miRSponge: a manually curated database for experimentally supported miRNA sponges and ceRNAs. Database 20152015, doi: 10.1093/database/bav098.

7. Tan JY, Sirey T, Honti F, Graham B, Piovesan A, Merkenschlager $M$, et al. Corrigendum: Extensive microRNAmediated crosstalk between IncRNAs and mRNAs in mouse 
embryonic stem cells. Genome Res 201525, doi: 10.1101/ gr.196568.115.

8. Karreth FA, Pandolfi PP. ceRNA crosstalk in cancer: when ce-bling rivalries go awry. Cancer Discov 2013; 3: 1113-1121, doi: 10.1158/2159-8290.CD-13-0202.

9. Wang J, Liu X, Wu H, Ni P, Gu Z, Qiao Y, et al. CREB upregulates long non-coding RNA, HULC expression through interaction with microRNA-372 in liver cancer. Nucleic Acids Res 2010; 38: 5366-5383, doi: 10.1093/nar/gkq285.

10. Xi L, Feber A, Gupta V, Wu M, Bergemann AD, Landreneau $\mathrm{RJ}$, et al. Whole genome exon arrays identify differential expression of alternatively spliced, cancer-related genes in lung cancer. Nucleic Acids Res 2008; 36: 6535-6547, doi: 10.1093/nar/gkn697.

11. Benjamini Y, Drai D, Elmer G, Kafkafi N, Golani I. Controlling the false discovery rate in behavior genetics research. Behav Brain Res 2001; 125: 279-284, doi: 10.1016/S01664328(01)00297-2.

12. Osareh F, Khademi R, Rostami MK, Shirazi MS. Coauthorship Network Structure Analysis of Iranian Researchers' scientific outputs from 1991 to 2013 based on the Social Science Citation Index (SSCI). Collnet J Scientomet Informat Manag 2014; 8: 263-271, doi: 10.1080/09737766.2014. 1015301.

13. Otte E, Rousseau R. Social network analysis: a powerful strategy, also for the information sciences. Journal of information Science 2002; 28: 441-453.

14. Wang C, Jiang W, Li W, Lian B, Chen X, Hua L, et al. Topological properties of the drug targets regulated by microRNA in human protein-protein interaction network. J Drug Target 2011; 19: 354-364, doi: 10.3109/1061186X. 2010.504261

15. Wolfe AW. Social Network Analysis: Methods and Applications. BMS Bull Sociol Methodol 1997; 24: 136-137.

16. Huang da W, Sherman BT, Lempicki RA. Systematic and integrative analysis of large gene lists using DAVID bioinformatics resources. Nat Protocols 2009; 4: 44-57, doi: 10.1038/nprot.2008.211.

17. Wang P, Ning S, Zhang Y, Li R, Ye J, Zhao Z, et al. Identification of IncRNA-associated competing triplets reveals global patterns and prognostic markers for cancer. Nucleic Acids Res 2015; 43: 3478-3489, doi: 10.1093/nar/gkv233.

18. Wang G, Chen H, Liu J. The long noncoding RNA LINC01207 promotes proliferation of lung adenocarcinoma. Am J Cancer Res 2015; 5: 3162.

19. Jones PA, Baylin SB. The fundamental role of epigenetic events in cancer. Nat Rev Genet 2002; 3: 415-428, doi: 10.1038/nrg962.

20. Li B, Carey M, Workman JL. The role of chromatin during transcription. Cell 2007; 128: 707-719, doi: 10.1016/j.cell. 2007.01.015.

21. Groth A, Rocha W, Verreault A, Almouzni G. Chromatin challenges during DNA replication and repair. Cell 2007; 128: 721-733, doi: 10.1016/j.cell.2007.01.030.

22. Watanabe $\mathrm{Y}$, Toyota $\mathrm{M}$, Kondo $\mathrm{Y}$, Suzuki $\mathrm{H}$, Imai $\mathrm{T}$, Ohetoyota $\mathrm{M}$, et al. PRDM5 identified as a target of epigenetic silencing in colorectal and gastric cancer. Clin Cancer Res 2007; 13: 4786-4794, doi: 10.1158/1078-0432. CCR-07-0305.

23. Takahashi H, Murai Y, Tsuneyama K, Nomoto K, Okada E, Fujita $\mathrm{H}$, et al. Overexpression of phosphorylated histone $\mathrm{H} 3$ is an indicator of poor prognosis in gastric adenocarcinoma patients. Appl Immunohistochem Mol Morphol 2006; 14: 296, doi: 10.1097/00129039-200609000-00007.

24. Broeck AVD, Brambilla E, Morosibilot D, Lantuejoul S, Brambilla C, Eymin B, et al. Loss of histone H4K20 trimethylation occurs in preneoplasia and influences prognosis of non-small cell lung cancer. Clin Cancer Res 2008; 14: 7237-7245, doi: 10.1158/1078-0432.CCR-08-0869.

25. Urrego D, Tomczak AP, Zahed F, Stuhmer W and Pardo LA. Potassium channels in cell cycle and cell proliferation. Philos Trans R Soc Lond B Biol Sci 2014; 369: 20130094, doi: 10.1098/rstb.2013.0094.

26. Eymin B, Gazzeri S. Role of cell cycle regulators in lung carcinogenesis. Cell Adh Migr 2010; 4: 114, doi: 10.4161/ cam.4.1.10977.

27. Harbour JW, Luo RX, Dei SA, Postigo AA, Dean DC. Cdk phosphorylation triggers sequential intramolecular interactions that progressively block $\mathrm{Rb}$ functions as cells move through G1. Cell 1999; 98: 859, doi: 10.1016/S0092-8674 (00)81519-6.

28. Meuwissen R, Linn SC, Linnoila RI, Zevenhoven J, Mooi WJ, Berns A. Induction of small cell lung cancer by somatic inactivation of both Trp53 and Rb1 in a conditional mouse model. Cancer Cell 2003; 4: 181-189, doi: 10.1016/S15356108(03)00220-4.

29. Cai J, Wu J, Zhang H, Fang L, Huang Y, Yang Y, et al. miR186 downregulation correlates with poor survival in lung adenocarcinoma, where it interferes with cell-cycle regulation. Cancer Res 2013; 73: 756-766, doi: 10.1158/00085472.CAN-12-2651.

30. Watanabe M, Maemura K, Oki K, Shiraishi N, Shibayama $Y$, Katsu K. Gamma-aminobutyric acid (GABA) and cell proliferation: focus on cancer cells. Histol Histopathol 2006; 21: $1135-1141$.

31. Wodarz A, Nathke I. Cell polarity in development and cancer. Nat Cell Biol 2007; 9: 1016-1024, doi: 10.1038/ ncb433.

32. Fu Y, Biglia N, Wang Z, Shen Y, Risch HA, Lu L, et al. Long non-coding RNAs, ASAP1-IT1, FAM215A, and LINC00472, in epithelial ovarian cancer. Gynecol Oncol 2016; 143: 642649, doi: 10.1016/j.ygyno.2016.09.021.

33. Shen Y, Katsaros D, Loo LW, Hernandez BY, Chong C, Canuto EM, et al. Prognostic and predictive values of long non-coding RNA LINC00472 in breast cancer. Oncotarget 2015; 6: 8579-8592, doi: 10.18632/oncotarget.3287.

34. Salih HR, Nüssler V. Commentary: Immune escape versus tumor tolerance: how do tumors evade immune surveillance? Eur J Med Res 2001; 6: 323-332.

35. Kübler K, Arndt PF, Wardelmann E, Landwehr C, Krebs D, Kuhn W, et al. Genetic alterations of HLA-class II in ovarian cancer. Int J Cancer 2008; 123: 1350-1356, doi: 10.1002/ ijc. 23624 .

36. Liu N, Zhang R, Zhao X, Su J, Bian X, Ni J, et al. A potential diagnostic marker for ovarian cancer: Involvement of the histone acetyltransferase, human males absent on the first. Oncol Let 2013; 6: 393-400, doi: 10.3892/ol.2013.1380.

37. Ruan W, Pei W, Feng S, Yuan X, Li Y. Long non-coding RNA small nucleolar RNA host gene 12 (SNHG12) promotes cell proliferation and migration by upregulating angiomotin gene expression in human osteosarcoma cells. Tumor Biol 2016; 37: 4065, doi: 10.1007/s13277-015-4256-7. 
38. Zhai W, Li X, Wu S, Zhang Y, Pang H, Chen W. Microarray expression profile of IncRNAs and the upregulated ASLNC04080 IncRNA in human endometrial carcinoma. Int J Oncol 2015; 46: 2125, doi: 10.3892/ijo.2015.2897.

39. Wang JZ, Xu CL, Wu H, Shen SJ. LncRNA SNHG12 promotes cell growth and inhibits cell apoptosis in colorectal cancer cells. Braz J Med Biol Res 2017; 50: e6079, doi: 10.1590/1414-431x20176079

40. Wang O, Yang F, Liu Y, Lv L, Ma R, Chen C, et al. C-MYCinduced upregulation of IncRNA SNHG12 regulates cell proliferation, apoptosis and migration in triple-negative breast cancer. Am J Translat Res 2017; 9: 533. 